\title{
Antioxidant Therapy in a Patient with Hyperprolinemia Type 1 Presenting with Mild Neuromotor Retardation and Speech Disturbance
}

\author{
Melike Ersoy $^{1} \cdot$ Semra Yılmaz ${ }^{2} \cdot$ Serdar Ceylaner $^{3}$ \\ Received: 1 January 2021 / Accepted: 16 March 2021 / Published online: 27 March 2021 \\ (C) Dr. K C Chaudhuri Foundation 2021
}

To the Editor: Hyperprolinemia type 1 (HP-1) is one of the two inherited metabolic disorders resulting from defects in the proline catabolic pathway [1]. The first step in proline catabolism is catalyzed by proline dehydrogenase (PRODH) that converts proline to proline 5-carboxylate (P5C). Symptoms of HP-1 include delayed psychomotor development, epilepsy, and neuropsychiatric symptoms [2].

A 4-y-old girl was admitted to the hospital with complaints of speech and neuromotor developmental delay and learning disability. The score of the intelligence quotient (IQ) test (Stanford-Binet Intelligence Scale) was 68. Routine biochemical tests were normal while amino acid analysis revealed high serum proline $892 \mu \mathrm{mol} / \mathrm{L}$ (n: 59-369). Brain magnetic resonance imaging (MRI) was normal. $P R O D H$ gene sequence analysis revealed c.1357C $>\mathrm{T}$ (p. Arg453Cys) homozygous mutation. We started antioxidant therapy with $100 \mathrm{mg} / \mathrm{d}$ coenzyme Q10 and B complex (B1 + B2 + B6 + B12), $500 \mathrm{mg} / \mathrm{d}$ vitamin $\mathrm{C}$, and $500 \mathrm{mg} / \mathrm{d} \mathrm{L}$-carnitine. The proline values in the third and sixth months of treatment were 273 and $400 \mu \mathrm{mol} / \mathrm{L}$, respectively, almost falling to normal limits. We ceased antioxidant therapy for one month and observed that the proline levels gradually increased to the levels of diagnosis range. Speech impairment increased and fine motor skills (e.g.,

Melike Ersoy

zeynepcey@hotmail.com

1 Department of Pediatrics, Division of Pediatric Metabolic Diseases, Bakirkoy Dr. Sadi Konuk Training and Research Hospital, University of Health Sciences, 34180 Istanbul, Turkey

2 Department of Pediatric Child and Adolescent Psychiatry, Bakirkoy Dr. Sadi Konuk Education and Research Hospital, Istanbul, Turkey

3 Intergen Genetics Center, Ankara, Turkey writing) was impaired dramatically during periods of cessation of treatment. A significant difference and an obvious negative correlation between with and without antioxidant treatment observed $(p<0.005 ; \mathrm{r}=-0.249)$. At 69 mo of treatment, IQ assessment showed a verbal score of 70, a performance score of 95 , and a full-scale IQ score of 81 . Her speech disturbance was improved.

Recent studies mentioned that low PRODH causes mitochondrial dysfunction by affecting electron transport chain [3]. PRODH binds directly to coenzyme Q1 and supports respiration independent of Complex I and II activity [4]. We could suggest that antioxidant therapy could serve as one of the potential therapy in HP-1, however, long-term multicentric experience may be pooled before concluding.

\section{Declarations}

Conflict of Interest None.

\section{References}

1. Afenjar A, Moutard ML, Doummar D, et al. Early neurological phenotype in 4 children with biallelic PRODH mutations. Brain Dev. 2007;29:547-52.

2. Di Rosa G, Pustorino G, Spano M, et al. Type I hyperprolinemia and proline dehydrogenase (PRODH) mutations in four Italian children with epilepsy and mental retardation. Psychiatr Genet. 2008;18:402.

3. van de Ven S, Gardeitchik T, Kouwenberg D, Kluijtmans L, Wevers R, Morava E. Long-term clinical outcome, therapy and mild mitochondrial dysfunction in hyperprolinemia. J Inherit Metab Dis. 2014;37:383-90.

4. Hancock CN, Liu W, Alvord WG, Phang JM. Co-regulation of mitochondrial respiration by proline dehydrogenase/oxidase and succinate. Amino Acids. 2016;48:859-72.

Publisher's Note Springer Nature remains neutral with regard to jurisdictional claims in published maps and institutional affiliations. 